\title{
Los viajes de los “dementes" del Provincial de Madrid durante la Guerra Civil (1936-1939).
}

The journeys of "mental illness" from Provincial Hospital of Madrid during The Spanish Civil War (1936-1939).

Olga Villasante a.

${ }^{a}$ Psiquiatra. Unidad de Psiquiatría. Hospital Severo Ochoa. Leganés (Madrid)

Correspondencia: Olga Villasante (ovillasante.hsvo@salud.madrid.org)

Recibido: 19/01/2010; aceptado: 25/04/2010

RESUMEN: El objetivo de este trabajo es conocer los traslados de pacientes psiquiátricos desde el Hospital Provincial de Madrid a diversas instituciones durante la Guerra Civil española (1936-1939). El material utilizado procede, fundamentalmente, del fondo documental del Archivo Regional de la Comunidad de Madrid (XII-1936 a IX-1938).

La necesidad de evacuar la Clínica Provincial de Madrid de enfermos crónicos les llevó a largos viajes hasta establecimientos psiquiátricos en la costa mediterránea como los de Alicante, Murcia o Almería, donde quedaron internos los dementes. Sin embargo, estas medidas no fueron suficientes y, posteriormente, se realizaron traslados al Instituto Psiquiátrico de Alcalá de Henares y a Saelices (Cuenca) e, incluso, fue preciso utilizar instituciones religiosas como en Almagro o un balneario real como La Isabela en Guadalajara.

PALABRAS CLAVE: Guerra Civil, traslados pacientes psiquiátricos, Hospital Provincial Madrid, Instituto Psiquiátrico de Alcalá, balneario La Isabela.
ABSTRACT: The aim of the paper is to know the movements of psychiatric patients from the Provincial Hospital of Madrid to different psychiatric and no-psychiatric institutions during the Spanish civil war (1936-1939).

The documental material referred to patients' movements was found in the Regional File of the Community of Madrid (XII-1936 a IX-1938). First, mental patients must travel long distances to the Mediterranean coast to Psychiatric institutions in Alicante, Murcia or Almeria. They also went to Psychiatric Institute of Alcalá de Henares or to Saelices (Cuenca), along 1937 and 1938. It was also necessary to use, enclosed religious institutions as Almagro's one and a real resort as The Isabela in Guadalajara.

KEYWORDS: Spanish civil war, movements of psychiatric patients, Hospital Provincial de Madrid, Psiquiatric Institute Alcalá, balneario la Isabela.

\footnotetext{
${ }^{1}$ Este trabajo se ha realizado en el marco del Proyecto de Investigación financiado por el Ministerio de Ciencia e Innovación, HAR08-04899-C02-01. E-mail de contacto: ovillasante.hsvo@salud.madrid.org
}

\section{AGRADECIMIENTOS}

Quería agradecer la amabilidad del personal de los Archivos consultados que me han prestado su ayuda y, especialmente, a Eustaquio Jiménez Puga del Archivo Municipal de Almagro. Por otra parte agradecer la información aportada personalmente por doña Luisa Bulnes, bisnieta de José Esquerdo y Zaragoza, y doña Teresa Viejo, periodista y autora de La memoria del agua. 


\section{Introducción}

Si la bibliografía general sobre la Guerra Civil española es hoy inabarcable y, casi a diario se siguen engrosando la literatura, ésta se vuelve considerablemente más escasa si tratamos de buscar trabajos que consideren aspectos médico-sanitarios de la contienda $(1,2)$. De hecho, salvo algunos trabajos aislados sobre los psiquiatras y la guerra $(3,4)$ los estudios sobre instituciones manicomiales, en general, se interrumpen al comenzar la guerra, ya que la excepcionalidad de la contienda civil provocó, entre otras cuestiones, destrucción de fuentes, inaccesibilidad a algunos fondos y, sobre todo, dificultades para abordar este período sin un marcado sesgo político. La silenciada guerra que siguió librándose, durante décadas, entre los derrotados republicanos y los victoriosos nacionalistas de algún modo, estableció un pacto de silencio y olvido prolongado, incluso, durante la transición democrática y que sólo ha empezado a romperse cuando ha aumentado la perspectiva temporal respecto a la Guerra Civil $(5,6,7,8,9)$.

Prácticamente no se encuentran trabajos monográficos en la historiografía moderna que hagan referencia al discurrir de las instituciones sanitarias o a los pacientes institucionalizados durante dicho trienio a excepción de ciertas referencias sobre algunos establecimientos psiquiátricos en trabajos más generales $(10,11,12)$. En los últimos años, se han analizado las historias clínicas de los pacientes ingresados en el Hospital Psiquiátrico de Leganés durante el periodo de la Guerra Civil española (13) y, recientemente, en un dossier monográfico de Frenia se ha estudiado la nosografía psiquiatrica (14), la demografía y los movimientos de población (15) y las medidas terapéuticas aplicadas en el Manicomio nacional de Leganés durante el período 1931-1952 (16), incluyendo, por tanto, el período bélico.

El objetivo, en este trabajo, es conocer la población de enfermos mentales del Hospital Provincial de Madrid durante la Guerra Civil, para lo cual hemos consultado, principalmente el Archivo Regional de la Comunidad de Madrid. En la búsqueda se han hallado los libros de filiación de hombres (17) y mujeres (18), si bien estos se interrumpen en 1936 y no se retoman hasta el 1 de junio de 1939, por lo que el estudio no es todo lo pormenorizado que nos gustaría. No obstante, hemos hallado una interesante documentación que hace referencia a traslados de pacientes psiquiátricos a diversas instituciones psiquiátricas entre diciembre de 1936 y septiembre de 1938. Desconocemos si la referida documentación abarca la totalidad de los movimientos de pacientes ocurridos durante la guerra, pero dado el considerable volumen de éstos, nos parece interesante su análisis, ya que esta información había sido sólo analizada parcialmente en una ponencia presentada en las XXI Jornadas Nacionales de la AEN celebradas en Alicante en la primavera de 2007. En dicho trabajo, La asistencia psiquiátrica a la población civil: Madrid en la guerra, se mostraron diversos documentos referentes a los traslados de pacientes hallados en el Archivo Regional, sin 
ORIGINALES Y REVISIONES

embargo, el hallazgo de más documentación nos ha llevado a redactar este trabajo. Pretendemos pues, en este artículo, aportar nueva información sobre lo ocurrido con algunos de los enfermos mentales internados en el Hospital Provincial, que tuvieron que viajar hasta Alcalá de Henares, Guadalajara, Saelices (Cuenca), Almagro, Alicante, Murcia e, incluso, Almería para permanecer allí ingresados.

\section{Las decimonónicas salas de enajenados}

Las salas de enajenados del Hospital Provincial habían suplido, si bien precariamente, la necesidad de un centro psiquiátrico médico específico en Madrid para la atención de alienados. A mediados del siglo XIX estos pacientes se hallaban "en un reducido, lóbrego y asqueroso local situado al norte y en la planta baja del edificio... se veían cinco covachas, o madrigueras, denominadas con toda propiedad jaulas, sin duda por la esacta ( (ic) semejanza con las de la antigua casa de fieras del Retiro"(19). Si bien José Rodríguez Villargoitia (1811-1854) (20), había realizado, en 1846, un proyecto para la construcción de un nuevo Departamento su propósito no se materializó y en las salas de S. Isidro y Santa María de la Cabeza (número 55 y 46) del hospital provincial, como eran denominadas las salas de hombres y mujeres respectivamente, los pacientes se hallaban hacinados. En principio, concebidas para recibir pacientes enajenados durante un breve período de tiempo, la provisionalidad del ingreso podía alcanzar, en ocasiones, más de tres años, tal como se recoge en la Memoria del año 1874 (21). De ese modo, el Hospital podía convertirse en un "depósito subterráneo", en el que sólo algunos pacientes podían ser trasladados al Hospital Provincial de los Inocentes (el Nuncio) de Toledo, San Baudilio o al Manicomio de Leganés. En la citada Memoria se describía un local carente de condiciones higiénicas, situado en los sótanos del hospital, sin luz, sin agua, ni ventilación, difícilmente ajustable a las condiciones exigidas por los principios terapéuticos de la especialidad. Ya hace años que González Duro realizó un análisis de los libros de registro de las últimas décadas del siglo XIX, en el que recogía datos como el estado civil de los enfermos, el tipo de internamiento, el porcentaje de traslados o la estancia media. Los traslados, que se habían realizado desde el año 1876 a San Baudilio de Llobregat, empezaron a decrecer a partir del año 1883, año en el que se firmó el acuerdo con el Sanatorio de Ciempozuelos (22). El número de derivaciones a Leganés fue inferior, ya que, desde el año 1870 la población psiquiátrica en el Manicomio Nacional era el triple de la prevista (23). Resulta significativo señalar que, en el año 1885, además del descenso de la estancia media, tuvo lugar una reducción marcada en el número de ingresos, seguramente atribuible a la promulgación del Real Decreto del 19 de mayo de 1885 que regulaba los mecanismos de ingreso. Por otra parte, en el citado Decreto se con- 
templaron los departamentos de observación de dementes ubicados en los hospitales provinciales o municipales, donde los pacientes debían permanecer hasta que se les condujera a un manicomio para su reclusión definitiva. Así pues, descendió el número de ingresos y se modificó la denominación antigua de las "salas de enagenados" del Hospital Provincial, pero no contribuyó a la mejora de la asistencia.

Sin duda, ni la apertura de Leganés (24), ni de las instituciones privadas fue suficiente para aliviar el hacinamiento de un departamento de enajenados que resultaba un local masificado con escasas posibilidades de derivación. Las declaraciones de Gonzalo Rodríguez Lafora (1886-1971) en el diario España, en 1916, han sido frecuentemente citadas y muy ilustrativas: "Del departamento de alienados del Hospital Provincial de Madrid, sólo diremos que permanece en el mismo estado que en la época de su fundación (1748). Allí no existen baños para los agitados, a los que sujeta mediante camisas de fuerza, otro utensilio desterrado ya de todas partes. Cuando se empiezan a acumular los enfermos una monja ordena traslados en masa a otros manicomios (al de Ciempozuelos o al de Valladolid), y los enfermos llegan a estos últimos con el único diagnóstico de enajenación mental y sin ningún dato ulterior que sirva para diagnosticarlos científicamente. Aún reciente es la publicación de agresiones de sus guardianes a un alienado" (25).

En los libros de filiaciones del Hospital General aparecen nombres como el de José María Palomino, Jaime Vera y López (1858-1918), Jerónimo Balaguer y Francisco Ángel Nieto, con la mención de "Profesor que a la sazón visitaba este Departamento". Entre estos, habría que destacar el nombre de Jaime Vera vinculado desde estudiante a José $\mathbf{M}^{\mathrm{a}}$ Esquerdo (1842-1912) y al nacimiento del socialismo en España. Este médico que ha sido definido como el primer teórico del marxismo español, ocupó una plaza por oposición en el Hospital General desde 1884 y se le ha atribuido la fundación del gabinete de electricidad donde se realizó, por primera vez, electro-diagnóstico y electro-terapéutica. A finales de siglo, en una carta autógrafa de 1891 de Esquerdo, el célebre psiquiatra aparecía como "titular de la Sala de Observación de Enajenados". En dicho documento Esquerdo expone la dificultad para trasladar a los pacientes cuyos expedientes "dementes reclusos" estaban terminados y se queja, asimismo, del hacinamiento de enfermos. Esta masificación, ante la escasez de altas y la falta de traslados, fue señalada por el titular de la salas de dementes, que afirmó que se encontraban 87 enfermos en un espacio cuya capacidad era para 30 ó 40 plazas (26).

\section{Las vísperas y el estallido de la guerra en el Hospital Provincial}

En las décadas previas al estallido de la Guerra Civil, como es bien sabido, la psiquiatría española gozaba de su mejor momento histórico ya que existía un grupo 
ORIGINALES Y REVISIONES

de profesionales que habían constituido la llamada primera "psiquiatría Científica Española" (27). Hombres de la talla de Gonzalo R. Lafora (1886-1971), José Miguel Sacristán (1887-1957), Enrique Fernández Sanz (1872-1950) o César Juarros (1879-1942), por citar sólo algunos de ellos, que constituían la llamada generación de Archivos de Neurobiología se vieron atrapados en una contienda en la que perdieron sus trabajos, sus hogares y, en ocasiones, incluso, su vida. El conflicto bélico rompió en dos un proyecto progresista de atención psiquiátrica que se había comenzado a cimentar sobre diversas empresas científicas gestadas en los años veinte -la publicación periódica de Archivos de Neurobiología (28), la formación de la Asociación Española de Neuropsiquiatría o la creación de la Liga Española de Higiene Mental- y que culminó en el Decreto de Asistencia a enfermos mentales del 3 julio de 1931(29).

En los años previos a la guerra el valenciano José Sanchís Banús (1890-1932) se ocupaba del servicio de neuropsiquiatría que, según Valenciano Gayá previamente (30), había llevado de un modo un tanto descuidado, Paco Huertas, hijo de Huertas Barrero. Sanchís Banús, además, era profesor de psicopatología en la Escuela de Estudios Penales escribió la tesis doctoral Estudio médico-social del niño golfo (31). El fallecimiento repentino de éste, el 22 de julio de 1932, dejó vacante la jefatura del Servicio de Neuropsiquiatría, del Hospital Provincial. A la oposición convocada sólo aspiraron Rodríguez Lafora y José M. Villaverde (1888-1936). Los discípulos de Lafora se agruparon a su alrededor, mientras Villaverde tenía como amigo incondicional a Carlos González Bueno (1898-1984) -ambos vestían sombrero verde, propio de los monárquicos porque reunía las iniciales de «Viva El Rey De España» (32)- que, después de la Guerra Civil, fue presidente del Colegio de Médicos de Madrid, y de la Diputación Provincial de Madrid. Moya dice haber recogido dos versiones acerca de esta oposición; la primera expresa que la ganó brillantemente Lafora y que los amigos de Villaverde pretendieron que se dividiera el servicio en dos, uno para Lafora y otro para Villaverde (33). Parece ser, según esta versión, que Lafora no puso dificultad alguna, ya que según dijo, con unas pocas camas le bastaba para trabajar adecuadamente. La segunda versión, en cambio, explica que Villaverde, de verbo brillante, realizó unos ejercicios extraordinariamente llamativos por su calidad en la exposición, frente a Lafora, que estuvo muy premioso y lento, como lo era con frecuencia. El resultado de la oposición, que ganó Lafora, la discutieron revistas médicas como El Siglo Médico y La Medicina Ibera, a cuya redacción pertenecía Villaverde. El tribunal, constituido por José Mouriz Riesgo (1884-1934), José Miguel Sacristán, Gregorio Marañón (1887-1960), José Goyanes Capdevila (1876-1964) y Fernando Enríquez de Salamanca (1890-1966), solicitó a la Diputación la creación de una segunda plaza, que se concedió a Villaverde, vulnerando ciertamente la ley de Administración Local, que no admitía ampliación de plazas. Parece que Sacristán fue quien propuso la división del Servicio en dos independientes, con la plena aquiescencia de Enríquez de Salamanca. Apasionadamente, los discípulos de Lafora 
elevaron un recurso contencioso-administrativo, encabezado por Luis Valenciano, que se resolvió por caducidad en plena Guerra Civil, debido a la ausencia de la formalización de los denunciantes ante la Audiencia Territorial de Madrid (34). Sea cual fuere la situación, la realidad es que, desde entonces, el Servicio de Psiquiatría quedó duplicado, de modo que Lafora se encargó de la sección de mujeres y Villaverde de los hombres. La salomónica decisión, sin embargo, no evitó futuros enfrentamientos dialécticos entre ambos médicos tanto en prensa por sostener criterios clínico-terapéuticos diferentes y como en la pugna por la vacante dejada por Santiago Ramón y Cajal (1852-1934), en la Academia Nacional de Medicina.

Villaverde pertenecía a la Escuela Neurohistológica de Cajal y se había formado en psiquiatría clínica junto a Antonio Fernández Vitorio (1855-1920). Había traducido a Bleuler de quien había sido asistente en Zúrich después de la concesión de una beca de la JAE. El marcado pensamiento monárquico y conservador de Villaverde, así como de la declaración a la Causa General de su propia hermana sobre su militancia en opciones ultraderechistas: Renovación Española y Falange Española, fue, sin duda la causa de su detención el 29 de septiembre de 1936 y su traslado a la checa de Fomento. Su asesinato nunca ha podido confirmarse porque no apareció su cadáver y al finalizar la Guerra, el juzgado le consideró "desaparecido".

Por otra parte, Lafora que ocupaba la plaza en la sala de mujeres, al igual que Villaverde desde 1933, se ocupó de ambos departamentos (mujeres y hombres) desde primeros de agosto de 1936, tal como afirmaba en una carta debido a la desaparición de su colega (30). Lafora había participado en la lucha por la reforma psiquiátrica, siendo vicepresidente de la Liga Española de Higiene Mental, cuando esta se había constituido en 1927 -el primer presidente fue Santiago Ramón y Cajal (1852-1934). En 1931, fue presidente del Consejo Superior Psiquiátrico, académico de número y, en 1935, presidente de la AEN. Al contrario que Villaverde, Lafora presentó un compromiso político con las ideas republicanas que precipitó su salida el 24 se septiembre de 1936 de Madrid hacia una casa familiar en Benidorm. Parece que pensaba volver unos días después, sin embargo, dado el discurrir de la Guerra y la necesidad de proteger a su hermana Isabel, religiosa ursulina, dificultó el regreso de Lafora a la capital. La baja de éste y varios profesores más motivaron que, en los primeros días de octubre, la Diputación de Madrid ordenara su inmediata incorporación a través de los medios radiofónicos y prensa escrita como El Heraldo. A finales de año, por un decreto de Sanidad, los médicos del Provincial no incorporados quedaron definitivamente retirados del servicio. Posteriormente Lafora regresó para recoger algunos libros, ya que, entonces tenía planes de quedarse en Valencia y organizar un curso de psicopatología así como una serie de conferencias culturales bajo los auspicios del Ministerio de Instrucción Pública. A partir del verano de 1937 se ocupó del Hospital Neurológico Militar que se instaló en Godella (Valencia) en el que trabajó con Sacristán hasta que, a mediados de 1938, salió de España como exiliado, previa estancia de 
ORIGINALES Y REVISIONES

un breve período en Barcelona. Así pues, sin Lafora, ni Villaverde en el Provincial, Vaamonde Valencia, según López Anón, quedó a cargo del servicio hasta 1939, fecha en la que se hizo cargo Ramón del Portillo (López Anón, p.15). La clínica psiquiátrica de mujeres, sin embargo, estuvo a cargo de Bartolomé LLopis (1905-1966) lugar donde este psiquiatra adquirió numeroso material clínico para el estudio de los trastornos psíquicos secundarios a la pelagra. De hecho, más de un centenar de casos de psicosis pelagrosa que observó durante la guerra civil sirvió como material para diferentes artículos publicados entre 1940 y 1944 -tiempo durante el que trabajó como telegrafista por la depuración de posguerra-. Su trabajo dio origen a su tesis de la psicosis única, vertida en la Psicosis pelagrosa, la psicopatología general y la nosología psiquiátrica (1940) (35).

\section{Los pacientes psiquiátricos en el Madrid de la Guerra Civil}

Tal como ya hemos referido, en los libros de filiación del Hospital Provincial no constan los ingresos que tuvieron lugar en sus salas a partir de julio del 36 y estos libros no se retoman hasta el 1 de junio de 1939, sin embargo Dionisio Nieto (19401985) publicó algunos datos sobre este período. Nieto de amplia formación neurohistológica, que había trabajado bajo la dirección de Sanchís Banús y colaborado con Nicolás Achúcarro (1880-1918), describió en "psiquiatría y neurología de guerra" los efectos de la guerra en la patología mental. En este trabajo que publicó en la Revista de Sanidad de Guerra (36), el que fuera director de Ciempozuelos hasta febrero de 1937 describía el considerable aumento de los ingresos en los cinco primeros meses de la guerra. Para Nieto había que considerar dos factores difíciles de cuantificar, como fueron las condiciones angustiosas de la vida en la ciudad, dados los bombardeos especialmente duros en otoño de 1936, así como el considerable aumento de la población de Madrid a expensas de las ciudades evacuadas de los sectores occidentales del frente. A partir de diciembre, sin embargo se inició un descenso brusco de los ingresos, inferior a lo normal, que se acentuó en los meses sucesivos y, quizá, explicable por la disminución progresiva de la población de Madrid debida a la evacuación a las zonas de retaguardia (37).

Vaamonde Valencia, médico residente de Lafora que había sustituido a éste después de su marcha a la costa valenciana aparece en la estadística de la sección de hombres publicada por Gregorio Bermann (1894-1972) (38), como Dr. Bahamonde, "jefe de servicio". Bermann era un prestigioso psiquiatra argentino que había llegado a España a finales de 1936, como brigadista internacional y había organizado la clínica de neurosis de guerra del Hospital de Chamartin de La Rosa $(39,40)$. En su libro Las neurosis de guerra (1941) se recoge la comparación entre los ingresos que 
tuvieron lugar en el Hospital Provincial de Madrid en dos períodos: de Julio de 1935 a marzo de 1936 y de Julio de 1936 a marzo de 1937, correspondiendo este último período, precisamente, a los primeros nueve meses de guerra civil (41). Aparecía un claro aumento de los ingresos sin que llegase a duplicarse (de 383 a 651), sobre todo, a favor de las reacciones psicógenas, el alcoholismo, y las causas exógenas, mientras que las psicosis endógenas experimentaban una variación curiosa. Durante el segundo semestre de 1936 las psicosis endógenas constituyeron el 31 por ciento de los ingresos de hombres, cifra especialmente baja ya que, sólo la esquizofrenia, constituía aproximadamente el $40 \%$ de los ingresos en los establecimientos psiquiátricos. Dionisio Nieto que, en 1939, se exilió a México (42), planteó que parecía poco verosímil el descenso de las psicosis endógenas y que, quizá, algunas de éstas fueron diagnosticadas como psicosis reaccionales merced a las circunstancias especiales en que se manifestaron.

El aumento de determinadas patologías fue utilizado por Vallejo Nágera para relacionar el incremento de estas psicosis exógenas con "el período de apogeo del terror rojo" (43). Sin duda los asesinatos de los grupos de izquierda más radical debieron influir en la patología psiquiátrica, pero no se puede obviar el efecto de los bombardeos ocurridos durante el otoño de 1936 y, en general, la angustia vivida en la ciudad sitiada. De hecho, el aumento de las reacciones psicógenas y psicopáticas ya había sido observado en otras guerras como la Primera Guerra Mundial. José Miguel Sacristán, reprodujo en forma de gráfico una estadística de Bonhoeffer en la que se apreciaba el incremento de las reacciones psicógenas en el período 1914-1917, en un breve artículo publicado en la revista Madrid. Cuadernos de la cultura (44). Esta revista fue publicada, durante un año, en la Casa de la Cultura en Valencia, donde se refugiaron muchos intelectuales afines a la República. Incluía ensayos sobre una gran variedad de temas, desde filosofía o crítica literaria hasta ciencia popularizada y hay que resaltar las contribuciones artísticas de pintores como Arturo Souto, José Solana y el escultor Victorio Macho. Entre sus colaboraciones literarias se pueden encontrar originales de Antonio Machado, León Felipe, Emilio Prados, Manuel Azaña, T. Navarro Tomas, José Bergamín.

Asimismo en otras guerras europeas también se había observado el incremento del alcoholismo aunque resulta difícil establecer comparaciones porque la mayoría de las publicaciones se ocuparon de poblaciones militares y las circunstancias sociales en la guerra civil española no son superponibles a las de otros países. A la dificultad que conlleva analizar dichos datos se añade el hecho de que los diagnósticos señalados por Vaamonde no están agrupados en entidades nosológicas acordes a las categorías kraepelinianas, tal como era exigido desde la Sección de Psiquiatría e Higiene Mental. Aunque, a partir de la orden del 28 de noviembre de 1931 (45), se había solicitado que todos los establecimientos psiquiátricos enviaran una estadística general en la que se contabilizaran los ingresos de acuerdo los XIV grupos 
ORIGINALES Y REVISIONES

diagnósticos de Kraepelin, en la estadística del Hospital Provincial las enfermedades aparecen agrupadas bajo siete epígrafes: psicosis endógenas, reacciones exógenas, personalidades psicopáticas, alcoholismo, toxicomanías, otras cusas exógenas y una última categoría en la que aparecen "casos oscuros, sin trastornos y sin datos".

Los ingresos en la Hospital Provincial, sin duda, estuvieron también condicionados por lo que ocurrió en otras instituciones que absorbían, hasta entonces, pacientes psiquiátricos como el sanatorio de Esquerdo o aquél dirigido por Rodríguez Lafora que dejaron de prestar dicho servicio en los meses siguientes al estallido de la guerra. Por una parte, el 7 de noviembre, el mismo día que el gobierno de la República se trasladó a Valencia y se constituyó la Junta de Defensa que presidió el General Miaja, se evacuaba el Sanatorio Neuropático de Carabanchel Bajo. Este sanatorio de aproximadamente 40 camas, que tuvo una intentona de incautación, había quedado en manos de Valenciano Gayá y Luis Ortega después de la salida de Lafora a Valencia. Estos, ante el avance de las tropas nacionales que habían ocupado Leganés y Getafe el día 4 y, sólo dos días más tarde, habían llegado al Hospital Militar, Cuatro Vientos y Carabanchel Alto, decidieron ubicar los enfermos en diversas clínicas de Madrid. Sin embargo, el apremio de dichas clínicas obligó a un nuevo traslado y el día 14 de noviembre, gracias a la gestión de Ramón Alberca, director del Hospital Psiquiátrico de Murcia desde 1928, los pacientes fueron admitidos en dicha provincia (30).

Por otra, el 4 de diciembre el sanatorio de Esquerdo, también en Carabanchel, fue bombardeado después de que un artillero, para la celebración de Santa Bárbara, descargara una batería. De los cerca de dos centenares de pacientes que albergaba, parece que en el ataque falleció, únicamente, un paralítico general; así que los pacientes fueron evacuados a la clínica de Las Piqueñas, también sita en Carabanchel Alto. Posteriormente fueron trasladados a una finca propiedad de Vicente Álvarez Rodríguez de Villamil, y Luisa Esquerdo Sáez en San Martín de Valdeiglesias, hasta que el obispo de Avila les cedió un convento para alojar a los caballeros. Los médicos Álvarez Villamil -miembro del Partido Reformista y candidato de la Conjunció Republicano-Socialista por Alicante a las elecciones generales de 1914-, Juan Esquerdo -hijo de José $\mathrm{M}^{\mathrm{a}}$ Esquerdo-, sus respectivas familias y los pocos empleados que habían quedado cuidaron de ellos durante el resto de la guerra (46).

\section{El viaje al Mediterráneo}

El aumento de los ingresos en la sala de hombres del Hospital Provincial sobre todo, a expensas de las reacciones psicógenas, los alcoholismos y las causas exógenas se debió según Vaamonde a diferentes factores: las recidivas en enfermos mentales 
descompensados bajo la influencia de las emociones de guerra y del sitio a Madrid, las recaídas de los alcohólicos y el mayor número de oligofrénicos que las familias no podían sostener al carecer de medios. Bartolomé Llopis, encargado de la sección de mujeres, subrayó la gran dificultad para que los familiares respondieran a las llamadas de los jefes de servicio, siendo incluso necesario entregar a los pacientes en el domicilio (41). Así pues, el colapso económico, las condiciones sociales específicas, la menor capacidad de las familias de contención de oligofrenias, el incremento del alcoholismo y las reacciones psicógenas desbordaron la capacidad de las salas del Provincial.

A partir del 4 de Noviembre de 1936, momento en que las tropas franquistas llegaron a Leganés, se interrumpió una de las posibles vías de salida de los crónicos del Hospital Provincial a donde eran derivados antes de la guerra. En las décadas previas, si bien con una limitada capacidad, el Manicomio de Leganés recibía pacientes procedentes del Hospital Provincial. Sin embargo, una vez comenzada la guerra y a lo largo de la contienda no ingresó ni un solo paciente procedente de dichas salas (13).

Por otra parte, durante los siete primeros meses de la guerra, sólo fueron trasladados dos pequeños grupos de enfermos a Ciempozuelos (37), mientras que en las décadas previas era este manicomio el que absorbía la mayor parte de los crónicos del Hospital Provincial. Dionisio Nieto había sido nombrado delegado técnico interino de la Dirección General de Sanidad de ambos establecimientos de Ciempozuelos -el de mujeres y el de hombres-, después de que se decretara el 5 de agosto de 1936 la incautación de "la Subsecretaría de Sanidad y Beneficencia del Sanatorio Psiquiátrico de San José y del Manicomio de señoras" (47). Según Valenciano Gayá, José Miguel Sacristán, director de este centro desde 1919, fue eliminado sectariamente de éste y del Sanatorio de los Ángeles (30). Este último era una pequeña clínica que albergaba, antes de la guerra cerca de 20 pacientes denominada Clínica Neuropsiquiátrica de Nuestra Señora de los Ángeles cuyo director era, así mismo, Sacristán. De todos modos Nieto iba a permanecer poco tiempo, durante el cual fue preciso trasladar 19 enfermas al Hospital de Toledo ya que la institución fue bombardeada (48).

A partir del 6 febrero de 1937 este sanatorio de los Hermanos de San Juan de Dios fue dirigido por Antonio Vallejo Nágera (1889-1960) (49), jefe de los Servicios Psiquiátricos Militares de ejército franquista y, nuevamente, en marzo de 1937 y octubre de 1938, se registraron bombardeos sin víctimas. A lo largo del período bélico, en el Hospital de mujeres, únicamente, ingresaron 18 pacientes y reingresaron otros 19 , una cifra ciertamente baja para un hospital que, cuando estalló la guerra, contaba con 1229 enfermas. De hecho, Vallejo Nágera había recomendado, por prudencia, no internar enfermos en Leganés, ni Ciempozuelos lo que favoreció una aglomeración en otros manicomios. El nuevo director de la clínica psiquiátrica de Ciempozuelos consideraba que, a diferencia de la zona republicana, en la España nacional no habían 
ORIGINALES Y REVISIONES

aumentado significativamente los ingresos, teniendo en cuenta, además, que existía una disminución de las camas disponibles debido a la destrucción de los manicomios de Huesca, Teruel y Asturias (43). Efectivamente, en el manicomio de Leganés no se incrementaron el número de ingresos $\mathrm{y}$, durante el trienio bélico, ingresaron un total de 64 pacientes, cifra similar a la de períodos previos (13). En el mismo sentido, López Ibor afirmó que, durante la guerra civil española, se pudo confirmar el dogma de la inamovilidad de las psicosis, así como de las neurosis bien constituidas. Afirmaba, además, que era sorprendente el escaso número de reacciones psicógenas (histéricos) y su simplicidad, lo cual atribuía a las "modalidades raciales de nuestro pueblo y el tono heroico de la contienda" (50).

El Consejo Superior Psiquiátrico propuso a ambas direcciones de Sanidad Militar y Civil, que las clínicas próximas a los frentes convertidas en cívico-militares, entre las que se incluía la de Madrid, fueran evacuadas de los enfermos crónicos civiles y destinaran el 60 por ciento de sus camas a los ingresos por psicosis reactivas o psicosis endógenas iniciadas reactivamente y exógenas (51). Así pues la difícil comunicación con la zona sur, donde se hallaban Leganés y Ciempozuelos, obligó a pensar en traslados hacia el este de la península. De hecho, Valencia ya había sido el destino, de numerosos niños también evacuados de Madrid, como aquella expedición que salió, a finales de octubre de 1936, con mil niños acogidos en el Instituto de Puericultura, en el Colegio de la Paz y de las Mercedes. Estos niños se distribuyeron en diferentes colonias en La Mancha (Viso del Marqués) y, fundamentalmente, en la zona de Levante (Burjasost, Aldaya, Tabernes de Valldigna, Pueblo de Navajas, pueblo de Jacarilla...) (52).

Poco después de que salieran aquellos niños, el día 1 de diciembre de 1936 salieron 124 pacientes de ambos sexos del Hospital Provincial (53 mujeres y 71 varones), acompañados de 15 enfermeros (53). Si bien desconocemos los diagnósticos de las mujeres, sabemos que, entre los varones trasladados a Alicante se hallaban 30 esquizofrenias, 10 oligofrenias, 4 psicosis maníaco-depresivas, 8 demencias en las que se incluían enfermos de P.G.P, 3 pacientes alcohólicos, 9 psicosis reactivas, 4 psicopatías y 3 depresiones reaccionales. Si comparamos la proporción de estos diagnósticos con otros períodos o instituciones, es llamativa, efectivamente la elevada proporción de oligofrenias que casi alcanza el $15 \%$ de los trasladados o las psicosis reactivas que superan el $12 \%$ y fueron, prácticamente, inexistentes en épocas previas.

Precisamente, Bartolomé Llopis, firmó la relación de enfermas evacuadas el 1 de diciembre de 1936, de las que 10 se quedaron en la Casa de Beneficencia Provincial de Alicante y, dado que no existía sitio suficiente, 43 mujeres fueron al Manicomio Provincial de Murcia (53). José Fernández López, el enfermero responsable de la evacuación, le dirigió una carta que firmaban otras 14 personas, el 10 de diciembre, al presidente de la diputación en la que se detallaban los gastos de conducción de 123 enfermos (71 varones y 52 mujeres) a los Manicomios de Alicante y Murcia. El gasto 
de la evacuación, que ascendían a 936 pesetas, incluía las dietas de 5 enfermeros y 10 enfermeras (5 días a 10 pesetas cada uno), así como los gastos de manutención entre los que se detallaban algunos alimentos (pan, latas de escabeche, naranjas, filetes, leche y churros). Durante el viaje y, ante la gravedad de una paciente se requirió el alquiler de un automóvil para avisar a un médico, así como la utilización de un ambulancia en Albacete (53). La enferma, trasladada a la casa de socorro de Albacete, falleció y por eso probablemente en la relación firmada por Bartolomé Llopis aparece el nombre de 53 mujeres. Según se desprende de la nota realizada por el responsable de la evacuación

\section{Documento 1}

Carta dirigida al Presidente de la Diputación Provincial con los gastos de manutención del viaje a Alicante y Murcia.

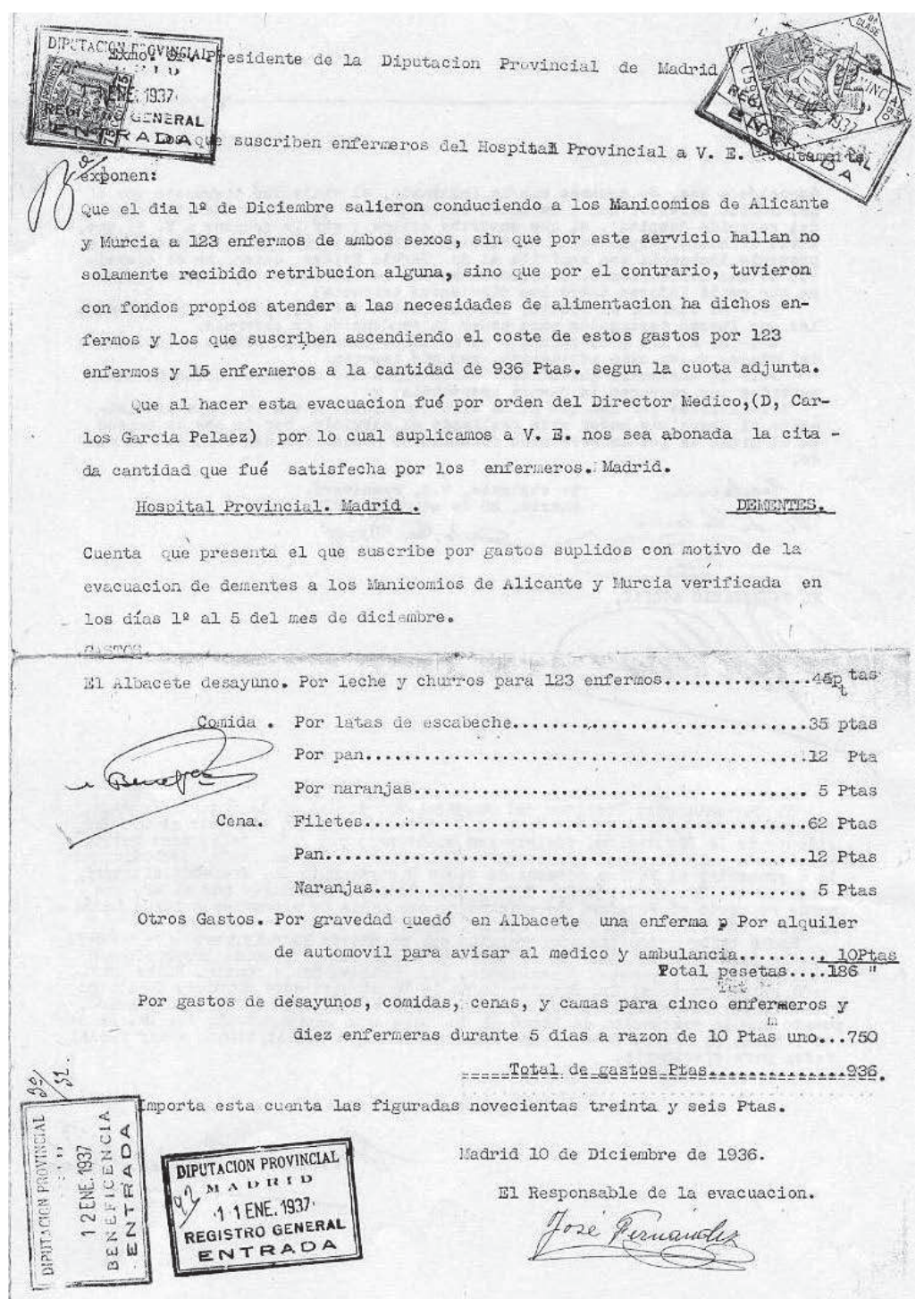
dos meses después aún no habían recibido la retribución correspondiente y, el 4 de febrero de 1937, el director médico del Hospital Provincial, Carlos García Peláez confirmaba que no se les había concedido "metálico alguno" y que, si bien él no había ordenado, ni autorizado el traslado, le parecían justificadas las partidas de gastos. El mismo director afirmó que el viaje se realizó a petición de Ezquerdo, cuyo representante fue el "Sr Cava" (documento I), y parece que la situación económica pudo quedar zanjada el día 25 de febrero cuando se realizó el abono (53). Sin embargo, el viaje de estos enfermos no llegó allí a su fin y, el 29 de enero de 1937, por insuficiencia de local en el manicomio Provincial de Murcia se trasladaron 40 varones al Manicomio Provincial de Almería (53). De hecho, el Hospital Psiquiátrico de 
ORIGINALES Y REVISIONES

Murcia fue uno de los que sufrió una gran sobrecarga y, durante el lustro 935-1939, el número de ingresos aumentó significativamente, de modo que casi se duplicó respecto al período previo 1930-1934, pasando de 397 a 748 (54).

De ese modo de aquellos 123 pacientes que salieron el 1 de diciembre de 1936 de la Clínica Psiquiátrica de Madrid hacia Alicante, allí sólo se quedaron 29 hombres y 10 mujeres. El resto aún viajaron hasta Murcia, donde 43 mujeres se instalaron en el Manicomio Provincial, mientras que los 40 varones restantes fueron trasladados al Manicomio Provincial de Almería. A esta lista habría que añadir la mujer que falleció en Albacete.

\section{De Alcalá de Henares a La Mancha}

En una carta de Lafora dirigida a Valenciano Gayá se afirmaba que existía un plan muy concreto para la evacuación de la clínica psiquiátrica del Hospital Provincial (13), sin embargo, como hemos visto no eran posibles las derivaciones ni a Leganés, ni a Ciempozuelos. Cerrada pues esta vía, el Hospital de San Juan de Alcalá se utilizó al año de comenzar la guerra, de modo que el 28 de julio de 1937, 30 varones que se hallaban en la salas de observación de dementes de Madrid se trasladaron a la población alcalaína (53).

Por otra parte, medio año después, el 15 de febrero de 1938 quedaron ingresadas 111 mujeres y 3 varones también en Alcalá, según una orden dictada por el Consejero Delegado de Sanidad. En esta relación se especificaba la edad, el origen y el diagnóstico de las enfermas, siendo la paciente más joven una chica oligofrénica de 14 años y la más anciana una mujer de 86 años con una demencia arterioesclerótica. Entre ellas el diagnostico más frecuente era el de esquizofrenia paranoide con 36 mujeres a las que se podrían sumar, una psicosis paranoide $\mathrm{y}$, al menos 3 hebefrenias; después seguían en frecuencia, 13 epilepsias, 9 oligofrenias, 8 cuadros demenciales que se diferencian entre senil y arteriosclerótica y también 8 cuadros relacionados con la lúes (pgp, tabes,..). Además se registran otros diagnósticos poco frecuentes como "psicópata”, "depresión endógena", "depresión angustiosa”, "síndrome de Ganser", "melancolía de la involución" o "pseudodemencia". De especial interés serían los casos de dos mujeres diagnosticadas de psicosis exógenas de 28 y 35 años, así como dos reacciones exógenas en pacientes de 28 y 40 años y una depresión reactiva de 44 años, sin embargo, dada la escasez de material clínico no se puede enriquecer la discusión generada en la época en torno a estos cuadros reactivos. Nuevamente desde el Hospital Provincial, el día 15 de abril de 1938 salieron otras 35 mujeres y el 21 de septiembre de 1938 se trasladaron 78 varones a Alcalá de Henares.

El Instituto Psiquiátrico de Alcalá, como es citado en alguna documentación era, junto a otros como la Casa de maternidad, el Instituto de Puericultura, el Hospital dermatológico provincial, el Hospital subalterno de Almagro o la escuela Hogar 
Maestro Ripoll, un establecimiento benéfico del Consejo Provincial de Madrid. La relación de pacientes, el 6 de diciembre de 1936, en el hospital de San Juan de Alcalá era de 91 pacientes varones, pero no todos ellos habían sido ingresos de la guerra, si no que había varones hospitalizados desde junio de 1929 (53).

En el momento que estalló la guerra, el nuevo Hospital Psiquiátrico Provincial de Alcalá de Henares, cuya construcción había comenzado en el año 1933, para una capacidad de 1672 enfermos, ampliable a 2000, estaba terminado y su inauguración prevista para septiembre de ese año (55). Lafora había colaborado junto al prestigioso arquitecto Baltasar Fernández Briz en su diseño, proponiendo una solución de pabellones situados en un terreno de abundante agua y rodeado de tierras de labor, pues se trataba de enfocar a los enfermos hacia una laborterapia de tipo agrícola. Sin embargo, la contienda impidió su inauguración y no fue utilizado como desahogo del Hospital de San Juan sino como cuartel, función que se mantuvo una vez finalizada la guerra. El centro, a pesar de no hallarse deteriorado, pasó al Ministerio del Aire donde se mantuvieron los cuarteles y con posterioridad al Ministerio del Ejército que lo dedicó a un Centro de Instrucción de Reclutas (33).

Así pues, el Hospital Psiquiátrico Provincial nunca fue utilizado para el fin que fue creado, pero el denominado "Sanatorio Psiquiátrico de Alcalá", "Instituto psiquiátrico" u "Hospital San Juan" albergó numerosos pacientes psiquiátricos. Esta institución alcalaína, de hecho sobrepasó su capacidad y, desde ésta y a lo largo de 1938, se realizaron diversos traslados a Almagro. Previamente fue preciso realizar gestiones con este municipio manchego y el día 30 de diciembre de 1937 quedaron resueltos los problemas para la instalación de dementes en la localidad. Esta cuestión acordada por el Consejero de Sanidad, García de la Serrana, fue publicada en El Socialista (56). La instalación de evacuados de Madrid venía ya practicándose previamente y sus gastos debían ser cubiertos por el Consejo Provincial de Madrid. Además, en esta población fue preciso establecer un taller para confeccionar ropa, según oficio del Administrador de los Servicios de Evacuación. Si bien en el Archivo Municipal de Almagro no existen documentos que lo certifiquen, algunos testimonios orales hacen pensar que se alojaran en el Convento de la Asunción de Calatrava (padres Dominicos) (57).

De ese modo el 15 de febrero de 1938 se trasladaron al denominado "sanatorio de Almagro", 75 varones y 9 mujeres; el 29 de abril, 28 mujeres y, al día siguiente, 56 varones; al mes siguiente, el 6 de mayo salieron 14 varones y el 13 de mayo de 1938 otros 16 hombres. La llegada de casi doscientos pacientes en tres meses llevó a que ese mismo verano el Hospital Subalterno Provincial instalado en Almagro dependiente del Consejo Provincial de Madrid, precisara la contratación de un jefe y profesionales sanitarios (enfermeros/as, psiquiatra...) y personal no sanitario como costureras. Por un parte, el 20 de agosto de 1938 fueron solicitados dos enfermeros para el departamento de dementes al Sindicato Único de Sanidad e 
ORIGINALES Y REVISIONES

Higiene (C.N.T-A.I.T) y a la U.G.T. de Madrid. Ambos sindicatos ofrecieron sendos afiliados para cubrir las vacantes, contratos remunerados con un jornal diario de 7,4 pesetas e internado (documento II). Poco después el mismo hospital, pero en este caso para el pabellón de mujeres dementes, tuvo que admitir provisionalmente a dos enfermeras porque dos de ellas habían dimitido. A las nuevas -María Rita Cuadra Gómez y Emilia Calero Castro- admitidas en octubre de 1938 se les asignó la cantidad de 1750 pesetas, inferior a las previas que cobraban 2000 pesetas. A fin

Documento 2

Solicitud de medicamentos.
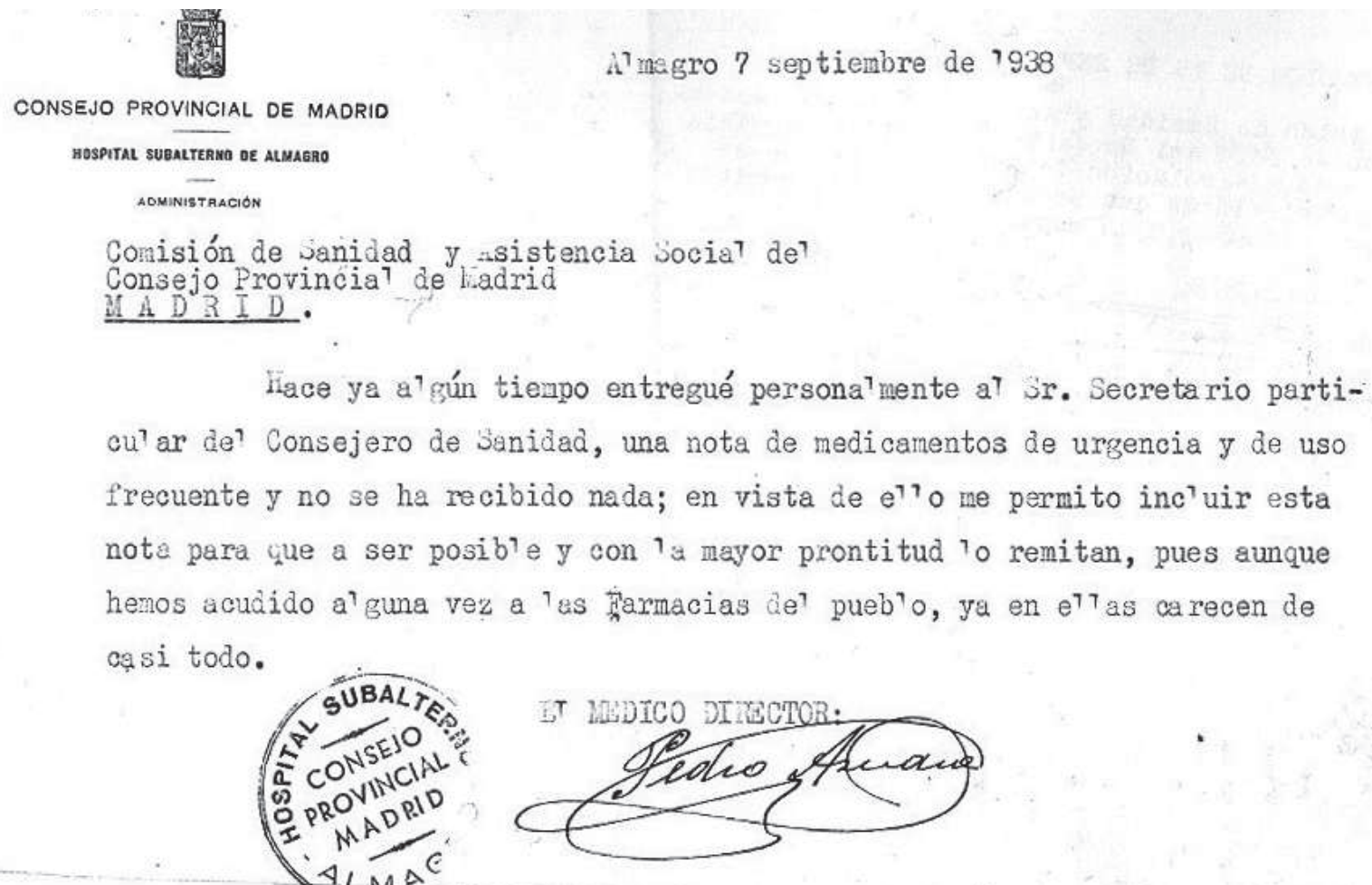

de año fue también preciso designar a Juan Martínez López, psiquiatra interino con el haber de 12.000 pesetas anuales, sueldo igual a quien ejercía de jefe. El abastecimiento de medicamentos no fue fácil, ya que, en septiembre de ese mismo año el médico director se quejaba de la escasez de éstos, ya que ni se podía abastecer en las farmacias del pueblo ni llegaban desde Madrid. (Documento II). No deja de sorprender que, en medio de la tragedia, de la pobreza y escasez de la guerra, en esta población de larga tradición teatral se inaugurara un teatro en el hospital. El día 9 de octubre de 1938 enfermos y empleados representaron "Esta noche me emborracho", pieza a la que fue invitado el mismo Presidente del Consejo Provincial 
Documento 3

Carta de invitación dirigida al Presidente del Consejo Provincial para informar e invitarle a una representación en el teatro del Hospital de Almagro.

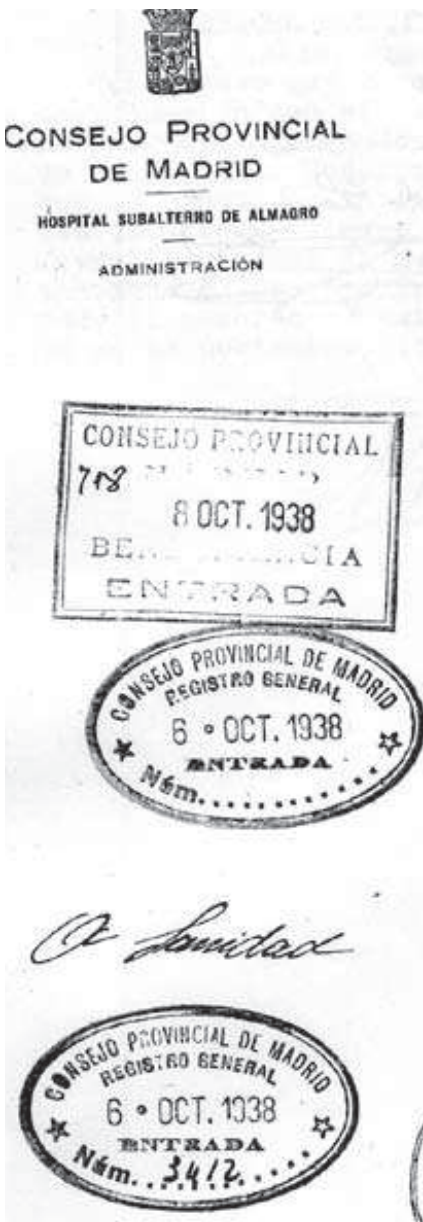

Tengo el gusto de poner en conocimiento de $\nabla$.3. que e' domingo dia 9 de' actua', se inaugurará $e^{T}$ Teatro de este Hospita', representándose 'a comedia " Esta noche me exborracho" interpretada por enfermos y emp’ eados de' Bstab? eciniento.

A comunioar'o a $\nabla$. $\mathrm{F}$. 'e invito, en nombre de 'os enfer mos y persona?, para que, si sus ocupaciones se 'o permiten, asista a esta representación que em-

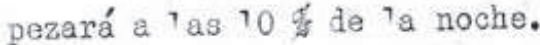
SUBAL magro 7 of octubre de 7938

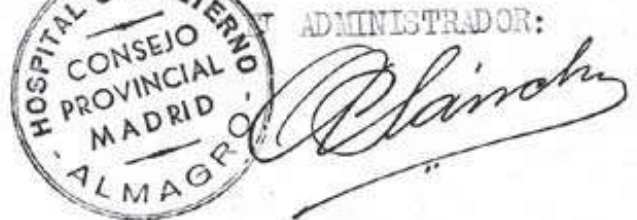

Gxcno. Sefior Presidente de' Consejo Provincia? de Madrid. de Madrid (4362/24)

(Documento III).

La improvisación de nuevos hospitales como el de Almagro durante la guerra, aún, no fue suficiente y se utilizaron lugares que, ni siquiera se han podido confirmar con documentación escrita o testimonios orales. Ejemplo de ello es una carta hallada en el mismo Archivo Provincial de Madrid en la que, si bien, se afirma que el 24 de julio de 1938 se trasladaron al manicomio de Saelices (Cuenca) 32 varones procedentes del Hospital Provincial, no se haya localizado la institución a pesar de haber realizado consultas en el Archivo de la localidad conquense, en el Archivo General de la Administración y en

el Archivo Histórico Provincial de la Junta de Comunidades de Castilla-La Mancha. La única referencia a instituciones sanitarias en dicho término Municipal fueron dos hospitales de sangre, uno de ellos instalado en Castillejos y otro en VillaPaz, un castillopalacio construido por la infanta Paz de Borbon, hija de Isabel II hacia el año 1898, y antigua residencia de verano de Alfonso XIII. Este hospital, utilizado por las Brigadas Internacionales, es citado en Take no prisoners, de Ray Durem, a quien Hughes incluyó en la antología New negro poets, ya que Durem se enamoró de una enfermera norteamericana que trabajó en este hospital instalado en Villa Paz. 
ORIGINALES Y REVISIONES

\section{Una residencia real para los dementes: el balneario de La Isabela}

Si bien no ha podido constatarse que Villa Paz acogiera a los dementes madrileños, si hay comprobación documental sobre las viejas instalaciones del ya decadente balneario La Isabela que acogió enfermos mentales durante la contienda. Construido sobre una pequeña península rodeada por el Guadiela al sureste de la provincia de Guadalajara y lindado con la parte septentrional de Cuenca, este pintoresco lugar, a ocho kilómetros de la población de Sacedón estaba asociado, desde la antigüedad, a un lugar de baños donde se asentó una población romana, y, después, musulmana. Dada, pues, dicha tradición termal Fernando VII, por mandato y capricho de la reina Isabel de Braganza, su segunda mujer, ordenó la construcción de una población de nueva planta en la orilla derecha del río Guadiela. Comenzadas las obras el 28 de enero de 1817 y declarado Real Sitio en 1826, el pequeño Versalles de la Alcarria, como fue conocido en la época, fue concebido a modo de ostentoso balneario, sin embargo la realidad sociopolítica no permitió finalizar el proyecto original. De hecho, este ejemplo de tardío urbanismo ilustrado fue bien diferente a la de los grandiosos proyectos de Carlos III marcándose, pues, ciertas diferencias con la categoría de otros Reales Sitios. El poblado de La Isabela conocido "Los Baños" estaba constituido por veintisiete manzanas de casas y unas cincuenta viviendas; un edificio destacado como cuartel para los guardias de Corps, además de otros servicios de posada, tienda, carnicería, horno de cocer, escuelas de niños y de niñas y una iglesia dedicada a San Antonio de Padua. La Casa Real, el más noble de sus edificios estaba destinada a ser la morada de los reyes que acudieran a la casa de Baños, a 150 metros separada de la residencia, sin embargo, las jornadas reales dejaron de tener como destino estos baños que constituyeron, a su vez, una obra benéfica y un lugar donde se desarrollaban actividades económicas que contribuyeron a su sostenimiento. El deterioro de los materiales, la escasa viabilidad económica y, definitivamente, la ley de Desamortización de 1865, lo separó definitivamente del patrimonio de la Corona, pero aún fue utilizado por la burguesía y la nobleza como establecimiento termal para aprovechar los efectos curativos de sus aguas -bebidas o en baño- (58). De hecho, sólo unos años antes de empezar la guerra, Gregorio Marañón exponía la bonanza de las aguas para enfermedades, entre otras, reumatológicas, dermatológicas, neurológicas o nerviosas, en Dominio, balneario y aguas de La Isabela: sobre la necesaria resurrección de los balnearios españoles (1931) (59).

Así pues la que había sido villa cortesana, adscrita al Socorro Rojo durante la guerra, se convirtió en el Sanatorio Psiquiátrico Nacional la Isabela para recibir a más de un centenar de pacientes del Hospital Provincial de Guadalajara a finales de 1936. Concebido, al principio, como Provincial recibió también pacientes de Teruel y de Madrid, como aquellos 75 varones que el 29 de diciembre de 1937 llegaron procedentes del Hospital General. Parece que, periódicamente, desde esta nueva institución 
en la que trabajó el médico Eduardo Varela de Seijas, se emitían informes dirigidos al Presidente del Consejo Provincial de Madrid del estado de dichos pacientes. Así, por ejemplo, el 1 de febrero de 1938 se describía que 46 de ellos no habían experimentado variación clínica alguna de los síntomas; 15 se encontraban adaptados, tranquilos y trabajando; 5 en la enfermería, 3 altas de permiso trimestral y 6 fallecidos (del 30.12.37 al 31.1.38). Nuevamente, el 8 de julio de 1938, el director del Sanatorio se dirigió al del Consejo Provincial de Madrid para informarle del estado de los 75 pacientes que habían sido trasladados seis meses antes, permaneciendo 48 de ellos en el mismo estado, 7 notablemente mejorados, 7 disfrutaban de una licencia trimestral y habían fallecido 13 de ellos (53). Esta alta mortalidad, debida, entre otras cuestiones, al hambre, a las condiciones higiénicas, a la escasez de tratamientos es relatada de forma novelada por Teresa Viejo, autora de La memoria del agua (60). Esta periodista, bien documentada si bien parte de su relato es ficción como el género lo exige, es una entretenida novela en la que se teje, entre otras intrigas, la historia de aquellos admitidos clandestinamente en la Isabela. Así pues esta villa termal organizada al modelo europeo en torno a dos calles geométricamente rectas, dos plazas y una extensa huerta rodeada de verja pudo acoger a "enajenados del frente", desertores de la derecha o religiosos que se confundieron entre los locos. Pero tampoco ese nuevo destino del balneario le iba a salvar de su destrucción, ya que el conjunto fue anegado, en 1957, por el embalse de Buendía y hoy sólo pueden ser contemplados los restos del balneario "La Isabela", cuando desciende el nivel de las aguas del pantano (61).

Además de la mortalidad recogida en los partes procedentes de La Isabella, en el Archivo Regional de la Comunidad de Madrid, también se han encontrado comunicaciones al Presidente de la Diputación Provincial con listas de pacientes fallecidos. Si bien no hay una serie consecutiva hemos hallado partes mensuales de marzo, abril, mayo, junio, agosto, octubre, noviembre y diciembre de 1937 y de enero a abril de 1938. En esta serie de 12 meses, que prácticamente corresponde a un año aparecen 100 hombres fallecidos y 80 mujeres (53), sin embargo es un dato, como ya hemos referido en tantos otros casos, de poco valor estadístico ya que no se pueden calcular las tasas al desconocer el número de ingresados en esa fecha.

\section{A modo de conclusión}

La Guerra Civil supuso una fractura en la sociedad civil española en todas las áreas y en la que la asistencia sanitaria, como es lógico, no se mantuvo al margen. La situación de excepcionalidad de la guerra provocó cambios en la atención psiquiátrica, que se practicó en condiciones de alta precariedad e, incluso, fue preciso desplazar a los pacientes desde su ciudad de origen. Del mismo modo que ocurrió con nume- 
ORIGINALES Y REVISIONES

rosa población, incluidos niños, los dementes fueron trasladados, a veces, a cientos de kilómetros desde sus domicilios, porque en la capital los pacientes psiquiátricos sólo podían ingresar en el Hospital Provincial y los médicos presentaban dificultades para darles de alta. Es difícil saber si estos lugares, más seguros por estar alejados del frente, podían ofertar a los pacientes unas mínimas condiciones sanitarias que, desde luego, tampoco estaban garantizadas en Madrid. Algunos, como hemos visto, se hacinaron en instituciones psiquiátricas, pero, en numerosas ocasiones se habilitaron establecimientos religiosos o residencias reales para los enfermos mentales en las que se planteaban, sin duda, problemas en el abastecimiento de medicamentos y en la contratación de personal sanitario. Si bien no ha podido comprobarse, probablemen-

Documento 4

Informe sobre los pacientes ingresados en La Isabella.

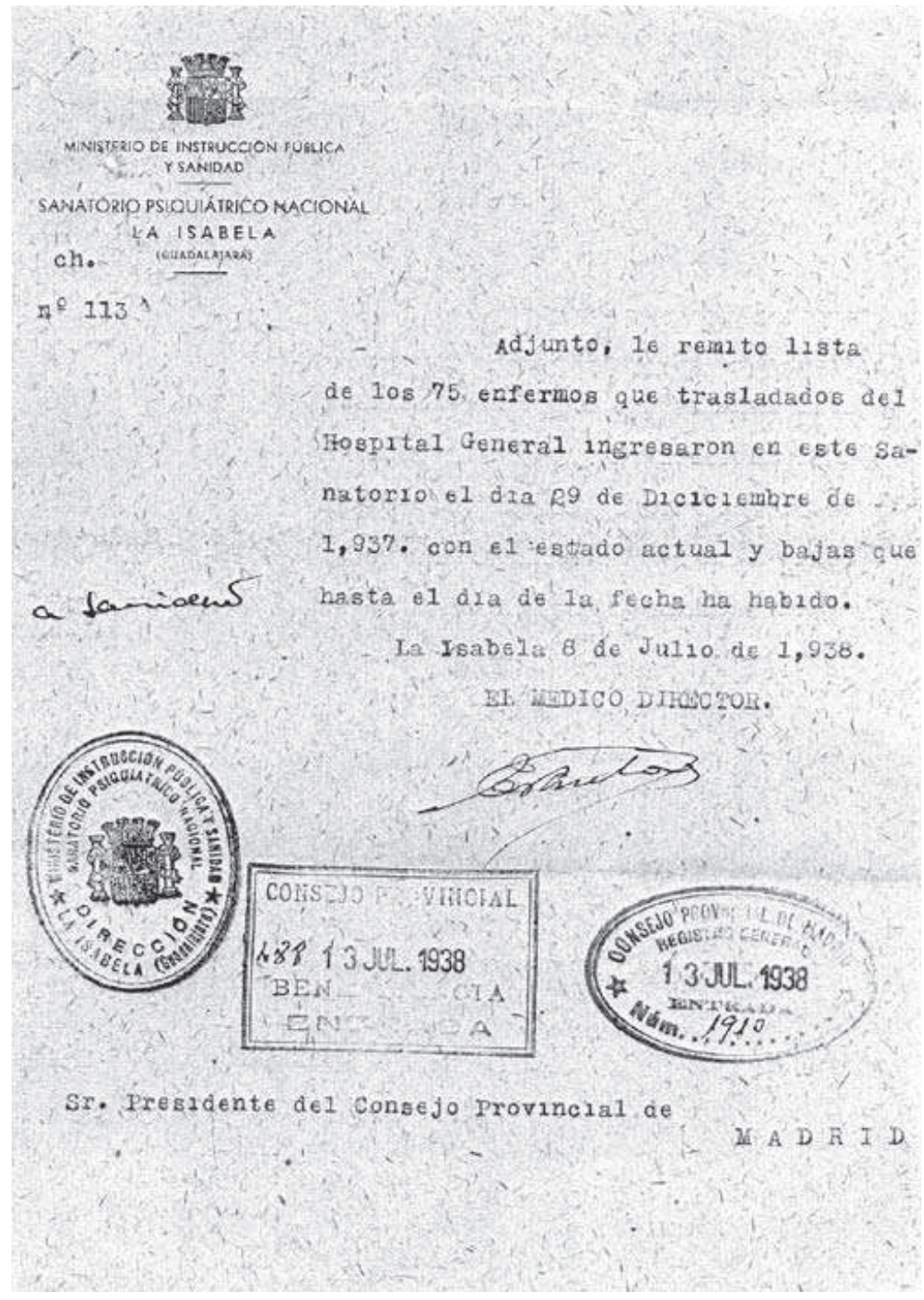

te las tasas de mortalidad eran altas en algunas de estas instituciones.

Las especiales condiciones de una Guerra Civil $y$, en el caso de España, la larga pervivencia de los ganadores ha condicionado la escasez de investigaciones relacionadas con la contienda, o en el caso de realizarse, éstas hayan presentado un significativo sesgo político que les resta valor científico, ya que en la posguerra sólo se oyeron las voces más afines al régimen franquista. Por ello las conclusiones que Vallejo Nájera sostenía sobre el menor número de admisiones en la zona nacional y el mayor índice de ingresos en las zonas controladas por el gobierno republicano, son difíciles de objetivar. Este mismo psiquiatra afirmó que se destruyeron archivos en la zona repu- 
blicana, pero, probablemente, no fueron las únicas destrucciones practicadas. La realidad es que en las numerosas consultas y búsquedas en archivos llevadas a cabo para este trabajo, me he encontrado con gran escasez de material documental. Los incendios, la destrucción, la sustracción voluntaria o, incluso, en alguna ocasión, el derecho al silencio que ha ejercido algún posible informante han generado un vacío historiográfico que, únicamente, será compensado por el empeño de aquellos que nos ilusiona la historia y de los investigadores e historiadores profesionales que, seguro, nos aportarán en un futuro aspectos aún hoy escondidos.

BIBLIOGRAFÍA:

(1) V.V.A.A., Los médicos y la medicina en la guerra civil, Madrid, Monografía Beecham, 1986.

(2) Larraz, P., León, P., Hospitales de guerra en Navarra (1936-1939). En Martínez-Pérez, J. et al. (ed.), La medicina ante el nuevo milenio: una perspectiva histórica, Murcia, Universidad de Castilla La Mancha, 2004:395-400.

(3) Carreras, A., Los psiquiatras españoles y la guerra civil. Medicina e Historia, 1986,13: $1-26$.

(4) Pérez Salas, P. La psiquiatría en la guerra civil: estudio bibliográfico, Realitat, 1992, 33: $42-46$.

(5) Iruela, L., Psiquiatría, psicología y armonía social: la vida y la obra de Emilio Mira y López, Barcelona, Universitat de Barcelona, 1993.

(6) Estalrich, J., Emilio Mira y la guerra civil española, Revista AEN, 1996, 16(59): 497-507.

(7) Simon LoRd, D., Médicos ourensáns represaliados na guerra civil e na posguerra. "Historias da longa noite de pedra”, Santiago de Compostela, Fundación 10 de marzo, 2002.

(8) CAPín, M., El valle de Dios. Una historia real y desgarradora, Ediciones MS-CYC, 2004.

(9) HuERTAS, R., La atención psiquiátrica a la población no combatiente durante la guerra civil española. En Campos, R. Villasante, O., Huertas, $R$. (eds.), De la "Edad de Plata” al exilio. Construcción y reconstrucción de la psiquiatría española, Madrid, Frenia, 2007:259-68.

(10) Comelles, J. M., Stultifera navis. La locura, el poder y la ciudad, Lleida, Editorial Milenio, 2006.

(11) HuERTAS, R., Spanish Psychiatry: The Second Republic, the civil war and the aftermat, International Journal of Mental Health, 2007, 35(4): 61-72.

(12) SALMÓN, F., HuERTAS, R., Unifying science against Fascism: Neuropsychiatry and medical education in the Spanish Civil War (1936-1939). En Kamminga, H., Geert, S. (eds.), Pursuing the unity of science: Scientific practice, ideology between the Great War and the Cold War, Aldershot, Ashgate Publishing, 2008.

(13) Villasante, O., Vazquez de la Torre, P., Tierno, R., La guerra civil en el Hospital psiquiátrico de Leganés: aproximación a un estudio de la población manicomial. En Martínez Pérez, Del Cura, M., Estévez, J., Blas, L. (Eds.), La gestión de la locura: conocimiento, prácticas y escenarios España, siglos XIX-XX), Cuenca, Ediciones Universidad de Castilla-La Mancha, 2008, 197-233. 
ORIGINALES Y REVISIONES

(14) VAZquez de La ToRRe, P., Nosografía psiquiátrica en el manicomio de Santa Isabel (19311952), Frenia, 2008, 8:69-96.

(15) TIERNo, R. Demografía psiquiátrica y movimientos de la población manicomial de la casa de dementes de Santa Isabel: 1931-1952, Frenia, 2008, 8: 97-130.

(16) Conseglieri, A. La introducción de nuevas medidas terapéuticas: entre la laborterapia y el electroshock en el manicomio de Leganés (1931-1952), Frenia, 2008, 8: 131-160.

(17) Archivo Regional Comunidad de Madrid. DIPUTACIÓN PROVINCIAL. HOSPITAL PROVINCIAL DE MADRID. Libro de registro de filiaciones hombres (tipo documental), signatura 900.730 .

(18) Archivo Regional Comunidad de Madrid. DIPUTACIÓN PROVINCIAL. HOSPITAL PROVINCIAL DE MADRID. Libro de registro de filiaciones mujeres (tipo documental), signatura 900.731.

(19) Torres, R., De la locura y los manicomios, La España Médica (IV), 1859, 162, p. 568.

(20) Rey, A., José Rodríguez Villargoitia (1811-1854), Revista AEN, 1984, 4, 10, pp. 264-274.

(21) Memoria del Hospital Provincial de Madrid, Madrid, Oficina Tipográfica, 1875, pp. 99-102.

(22) GonzÁlez Duro, E., Asistencia psiquiátrica madrileña a fines del siglo XIX, Medicina e historia, 1971, 51, p. 10.

(23) Delgado, M., Los veinte primeros años del Manicomio Modelo de Leganés (1852-1871), Asclepio, 1986, 38, p. 274.

(24) Villasante, O., Orden y norma en el Manicomio de Leganés (1851-1900): El discurrir diario del paciente decimonónico, Frenia, 2008, 8: 33-68.

(25) RodríGuez Lafora, G., Los manicomios españoles, España, 1916, 90: 8-10.

(26) López Anón, A., Historia de un Servicio, Cuadernos de Psiquiatría Madrileña, 1971,2 (7): $12-15$.

(27) Rodríguez Lafora, G., Spanish Psychiatry during the last decade, American J. Psychiatry, 1949, 105(12): 901-903.

(28) LÁZARO, J. Archivos de Neurobiología: los setenta y cinco años de la psiquiatría española, Archivos de Neurobiología, 1995, 58(1): 13-30.

(29) CAmpos, R., Huertas, R., Estado y asistencia psiquiátrica en España durante el primer tercio del siglo XX, Revista AEN, 1998, 18 (65), 99-108.

(30) Valenciano, L., El Dr. Lafora y su época, Madrid: Morata, 1977, p. 77.

(31) Huertas García-Alejo, R., Clasificar y educar: historia natural y social de la deficiencia mental, Madrid, CSIC, 1998.

(32) Rey González, A. Martí Boscá, J. V. Un monárquico y un anarquista. Debate sobre el psicoanálisis (1924-1928): José Ma Villaverde e Isaac Puente. En Campos, R. Villasante, O., Huertas, R. (eds.) De la "Edad de Plata" al exilio. Construcción y reconstrucción de la psiquiatría española, Madrid, Frenia, 2007, 73-94.

(33) Moya, G., Gonzalo R. Lafora. Medicina y cultura en una España en crisis, Madrid, UAM:85-9.

(34) Villasante, O., Rey, A., Martí, J. V., José Ma Villaverde: retrato de un desconocido, Medicina e Historia, 2008, 1.

(35) Del Cura, I. and Huertas, R., Alimentación y enfermedad en tiempos de hambre. España, 1937-1947, Madrid, CSIC, 2007 
(36) VAZQuez de LA TORRe, P, TIERNo, R., La literatura psiquiátrica durante la guerra civil española (1936-1939): "Archivos de Neurobiología", "Revista de Sanidad de Guerra" y "Revista Española de Medicina y Cirugía". En Campos, R. Villasante, O., Huertas, $R$. (eds.) De la "Edad de Plata" al exilio. Construcción y reconstrucción de la psiquiatría española, Madrid, Frenia, 2007:239-258.

(37) Nieto, D., Psiquiatría y Neurología de guerra. Revista de Sanidad de guerra, 1937, 1(5):182-193.

(38) Villasante, O., Gregorio Bermann y La neurosis de guerra en el Madrid de La guerra civil española, Temas de Historia de la Psiquiatría Argentina, 2009, 27,13-20.

(39) Villasante, O., La producción científica en torno a la neurosis de guerra. En Campos, R. Villasante, O., Huertas, R. (eds.), De la "Edad de Plata" al exilio. Construcción y reconstrucción de la psiquiatría española Madrid, Frenia, 2007, 179-200.

(40) Villasante, O. "War neurosis" in Spanish Civil War in Madrid (1936-1939), History of psychiatry, (en prensa).

(41) Bermann, G., Las neurosis en la guerra, Buenos Aires: Aniceto López, 1941, p. 52.

(42) Sacristán, C., En defensa de un paradigma científico. El doble exilio de Dionisio Nieto en Mexico. En Campos, R. Villasante, O., Huertas, R. (eds.), De la "Edad de Plata" al exilio. Construcción y reconstrucción de la psiquiatría española, Madrid, Frenia, 2007, 327-346.

(43) Vallejo Nágera, A., Psicosis de guerra, Madrid, Ediciones Morata, 1942, p. 32.

(44) SACRISTÁn, J. M., La guerra como causa de alteraciones psíquicas, "Madrid", Revista de la Casa de la Cultura de Valencia, 1937, 93-96.

(45) Gaceta de Madrid, 2 diciembre 1931.

(46) Esta información ha sido facilitada gracias a la amable colaboración de doña Luisa Bulnes, bisnieta de José Esquerdo y Zaragoza y actual presidenta de la Fundación Esquerdo.

(47) Gaceta de Madrid, 8 agosto 1936, 221, p. 1149.

(48) López de Lerma Peñasco, Díaz Gómez, M., Historia del hospital psiquiátrico Sagrado Corazón de Jesús, de Ciempozuelos, 1881-1989, Madrid, Fareso, 1991, pp. 217-218.

(49) Huertas, R., Los médicos de la mente. De la Neurología al psicoanálisis. Lafora, VallejoNágera, Garma, Madrid: Nivola, 2002.

(50) LóPez-IBOR, J. J., Experiencias psiquiátricas de Guerra, Revista Española de Medicina, 1938, 5:82-101.

(51) Rodríguez-Lafora, G., La Psiquiatría y la Neurología de Guerra y de la Revolución. Sus problemas y soluciones, Revista de Sanidad de Guerra, 1937, 1(4): 121-128.

(52) EsPina PÉREZ, P., Historia de la inclusa de Madrid, www.cervantesvirtual.com/servlet/ SirveObras/.../022401_041.

(53) Archivo Regional de la Comunidad de Madrid. Diputación Provincial. Sección de Beneficencia Signaturas por orden de citación: 4356/8, 4356/1, 4356/3, 4356/3, 4356/2.

(54) Valenciano Gayá, Parálisis general Progresiva, Diputación Provincial, Murcia, 1978, p. 36.

(55) Rodríguez Lafora, G., La asistencia al enfermo mental en las provincias españolas y la capital, Diario ABC, 22 de noviembre de 1961.

(56) El Socialista, 31 de diciembre 1937.

(57) La información sobre el lugar que ocupó el hospital ha sido aportada por Eustaquio Jiménez Puga, archivero municipal de Almagro. 


\section{ORIGINALES Y REVISIONES}

(58) Aguado Pintor, A., La Isabela, un nuevo Real Sitio para los monarcas del siglo XIX, Espacio, Tiempo y Forma. Serie VII; Historia del Arte, t. 15, 2002, 229-254.

(59) Marañón, G., Dominio, balneario y aguas de La Isabela: sobre la necesaria resurrección de los balnearios españoles, Madrid, 1931.

(60) VIEJo, T., La memoria del agua, Madrid, mr ediciones, 2009.

(61) García López, A., El Real Sitio de La Isabela y los baños de Sacedón, Guadalajara, Ediciones Bornova, 2008. 